\title{
Squeezed light generated by a microcavity laser
}

\author{
Daniel C. Kilper, Peter A. Roos, and John L. Carlsten \\ Department of Physics, Montana State University, Bozeman, Montana 59717
}

Kevin L. Lear

Sandia National Laboratories, P.O. Box 5800/M.S. 0603, Albuquerque, New Mexico 87185-5800

(Received 18 February 1997)

\begin{abstract}
Photon-number fluctuations $1.3 \mathrm{~dB}$ below the semiclassical shot-noise limit are observed in the output of a semiconductor microcavity laser. Although the laser oscillates in a single longitudinal mode, photon-number squeezed light is realized through nonclassical correlations between two orthogonally polarized, transverse laser modes. [S1050-2947(97)50705-0]

PACS number(s): 42.50.Dv, 42.55.Sa
\end{abstract}

When the direct generation photon-number squeezed light from an optical source was first demonstrated [1], this macroscopic quantum effect was scarcely measurable due to the debilitating effect of high optical losses. Shortly thereafter, the laser emerged as a natural solution to this problem. Yamamoto and co-workers demonstrated that the laser, previously thought to be limited to coherent-state output, can naturally emit photon-number squeezed light [2]. In particular, the semiconductor laser features the essential elements necessary to realize photon-number squeezed output, including high-efficiency operation at high pump rates and pumpnoise suppression through the drive current. Laser pump noise, which limits the low-frequency intensity noise on the output of a laser at high pump rates, can be suppressed below shot noise $[2,3]$, resulting in photon-number squeezed output. Recently, the collimated output of cryogenically cooled semiconductor quantum-well lasers demonstrated large (4.5 $\mathrm{dB})$ squeezing near levels expected from the device efficiency [4], and previous measurements suggest that the squeezing may reach limits associated with a laser's optical efficiency [5]. Indeed, during the past decade considerable progress has been made in understanding the quantum-noise processes of conventional semiconductor lasers $[2,4-6]$ and photon-number squeezed light has reached the predicted device efficiency limits. Despite this progress in the generation of photon-number squeezed light from conventional semiconductor lasers, the photon-number fluctuations previously observed from electrically pumped microcavity semiconductor lasers have been limited to an order of magnitude above shot noise [7].

Nevertheless, the microcavity laser has been proposed as an ideal source for photon-number squeezed light, with several important benefits over conventional semiconductor lasers. Microcavity devices are characterized by a cavity length matched to the wavelength of light. This extreme reduction in mode volume, potentially enhanced by cavity quantum electrodynamic effects [8], results in a significant enhancement of the fraction of spontaneous emission into the lasing mode. Ultimately suppression of all emission into nonlasing modes can result in squeezed output for all laser pump rates [9] (i.e., high efficiency without the need for stimulated emission). While this effect is not achieved in present devices, the small volume contributes to submilliampere threshold currents. The benefit of a low threshold is that the high pump rates necessary to generate squeezed output may be achieved at room temperature without damage to the laser. Typical semiconductor microcavity lasers also have high mirror reflectivities (>99\%) in order to build up gain over the short active region. Therefore, microcavity lasers operate in the good-cavity limit (with a finesse two orders of magnitude above conventional devices), which is the limit used in the standard quantum laser theory that predicts photon-number squeezed output [2].

One type of semiconductor microcavity laser, the verticalcavity, surface-emitting laser (VCSEL) diode, has several important differences from conventional semiconductor lasers. Longitudinal-mode competition noise is not present in these microcavity devices because they operate in a single longitudinal mode. In conventional devices, noise associated with the longitudinal modes is often dominant [6]. On the other hand, transverse-mode confinement is still being perfected in VCSELs, and emission is frequently observed in several transverse laser cavity modes [10]. The quantumnoise properties of multiple longitudinal modes have been studied in some detail, but the related behavior of multiple transverse modes has not been examined. Furthermore, in VCSELs the orthogonal polarization modes are nondegenerate (by $>1 \mathrm{GHz}$ ), and mode correlations, which are expected between linearly polarized modes from the selection rules in the quantum wells, can be distinguished from mode beating effects, whereas orthogonal polarization modes of a conventional laser are more nearly degenerate, and the behavior is complicated by birefringent mode mixing [4]. Thus current semiconductor microcavity lasers have properties, such as multi-transverse-mode operation (in a single longitudinal mode) and high cavity finesse, that have not been studied in the quantum regime using conventional devices. The observation of photon-number squeezed output from microcavity lasers provides insight into these aspects of quantum laser theory, and establishes that such sources are attractive to push the limits of nonclassical emission.

In this paper, we report photon-number squeezed output from a semiconductor microcavity laser. Measurements using both single-detector and balanced-detector configurations demonstrate photon-number fluctuations $1.3 \mathrm{~dB}(25 \%)$ below the semiclassical shot-noise limit. For drive-current levels at which squeezing is observed, the laser is found to be oscillating in the two lowest-order transverse modes, linearly po- 


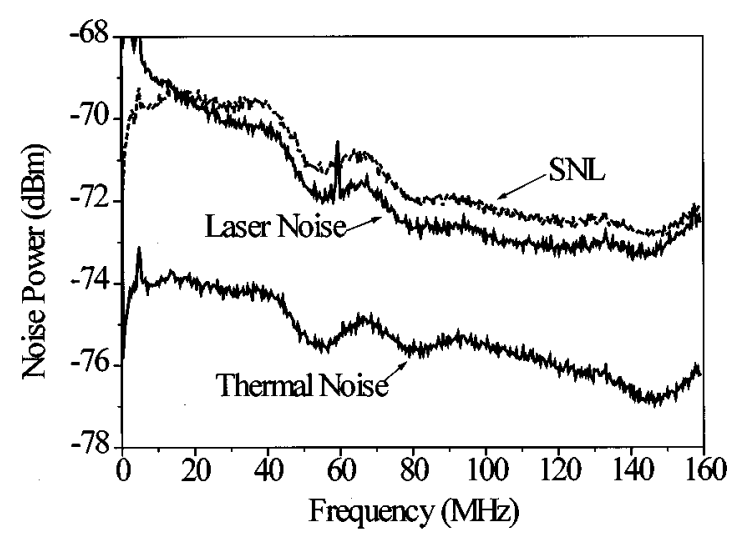

FIG. 1. Photocurrent noise power spectra of a microcavity laser biased at a pump rate of 11 (Laser Noise), the shot-noise level recorded using a red-filtered halogen lamp (SNL), and the background thermal noise (Thermal Noise).

larized along orthogonal polarization axes. Similar to the case for longitudinal modes, the individual transverse modes exhibit noise levels far above shot noise, while the combined fluctuations are below shot noise. These results provide clear evidence of quantum correlations between multiple transverse laser modes.

The device used in this experiment is an oxide-confined, vertical-cavity, surface-emitting laser [11]. The length of the AlGaAs laser cavity was grown to match the nominal lasing wavelength of $960 \mathrm{~nm}$. The lateral dimensions are defined by selectively oxidized layers of AlGaAs surrounding $\mathrm{a} \sim 3 \times 3-\mu \mathrm{m}^{2}$ aperture. These oxide layers provide index and gain guiding to the optical field inside the cavity. The active region is comprised of three 8-nm $\mathrm{In}_{0.2} \mathrm{Ga}_{0.8}$ As quantum wells. The bottom distributed Bragg reflector (DBR) mirror is $>99.9 \%$ reflective and the top (output) DBR mirror is $>99 \%$ reflective.

The photocurrent noise power spectrum obtained by directing the VCSEL output to a single Hamamatsu S1722-01 detector is shown in Fig. 1. A high (0.68) numerical aperture molded aspheric lens was used to adjust the laser spot size to fill the 4-mm-diam detector active region positioned $0.25 \mathrm{~m}$ from the laser, which corresponds to a rough collimation. The temperature of the device was stabilized using a thermoelectric cooler to eliminate potential temperature-dependent effects; however, similar results were obtained without temperature control. The photocurrent noise, which is a measurement of the photon-number fluctuations, was amplified by $40 \mathrm{~dB}$ and measured on a spectrum analyzer $(1-\mathrm{MHz}$ RBW, 3-kHz VF, 1-sec sweep). The laser noise trace shows the laser noise power spectrum at a drive current of 2.897 $\mathrm{mA}$ (12 times threshold, $I_{\mathrm{th}}=0.24 \mathrm{~mA}$ ) and a detector current of $1.056 \mathrm{~mA}$. The shot-noise trace was recorded by illuminating the detector with a red-filtered halogen lamp (a source of classical light) with a $1.042-\mathrm{mA}$ photocurrent. The background amplifier thermal noise level also shown has not been subtracted from the other traces. The laser output photon-number fluctuations are clearly observed to fall below shot noise for frequencies above $20 \mathrm{MHz}$. A 171-point average from 60 to $114 \mathrm{MHz}$, after subtraction of the thermal noise trace, yields a laser noise level $1.3 \mathrm{~dB}$ below shot noise.

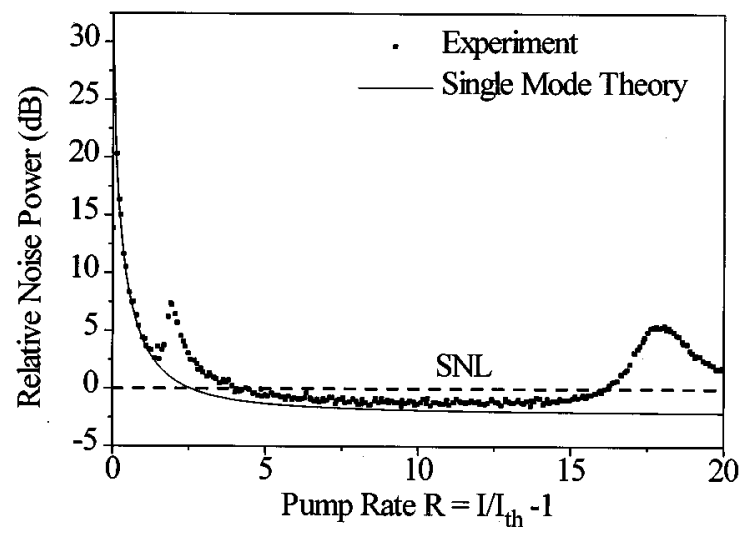

FIG. 2. Photocurrent noise power at $97.5 \mathrm{MHz}$, normalized to the shot-noise level, as a function of laser pump rate (squares); the solid curve is calculated from the single-mode quantum-Langevin equations.

These single-detector noise measurements compared against a reference field were carefully tested because of potential sensitivity of the detector response to variations in illumination spot size, frequency, and intensity distribution [12]. The detectors used in this experiment have been thoroughly characterized in previous work [12], and found to be linear at the intensity levels used in this work. Measurements of the particular detectors in this experiment demonstrate similar linearity. Furthermore, measurements taken with a larger-area Hamamatsu S3994 detector, which has superior linearity, also demonstrate squeezing in agreement with the S1722 detectors. However, the limited frequency response (high-frequency cutoff near $60 \mathrm{MHz}$ ) restricts the range of frequencies over which squeezing is observed. Varying the laser spot size on the S1722 detector revealed a constant response until a tight focus was formed on the detector at which point a $0.5-\mathrm{dB}$ drop in noise power was observed. These measurements indicated that spot-size-dependent saturation is only significant when the beam is at a focus. Care was also taken to ensure that optical feedback to the laser was not influencing the results.

Strong confidence in a squeezed light measurement is achieved by using a balanced homodyne detector. Such devices allow for a simultaneous measurement of the laser noise and shot noise under identical conditions (spot size, etc.). The presence of polarization-dependent noise, as described below, requires that a polarization-insensitive beam splitter be used in the balanced detector. For this purpose, a hybrid cube beam splitter was used to provide roughly equal splitting between polarizations. Measurements using a balanced homodyne detector confirmed the observation of squeezing in this experiment. The balanced detector shotnoise level was found to match the shot-noise level of a halogen lamp to within $0.1 \mathrm{~dB}$. The single-detector measurements are preferred in this case, however, because the balanced detector thermal noise level is twice the singledetector level, and residual polarization effects can be ruled out for a single detector.

The laser noise as a function of pump rate $\left(I / I_{\mathrm{th}}-1\right)$ is shown in Fig. 2, and compared with the single-mode, quantum laser theory. The experimental data were obtained by stepping the drive current in $0.02-\mathrm{mA}$ steps from 0 to 5.0 


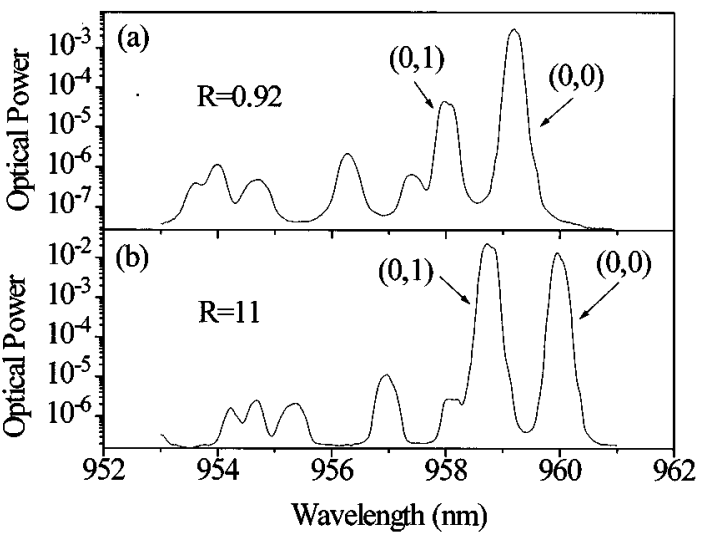

FIG. 3. Optical power spectra at two different pump rates: (a) $R=0.92,(0,0)$ transverse mode dominates by two orders of magnitude; (b) $R=11,(0,0)$ mode and $(0,1)$ mode with roughly equal amplitudes (0.1-nm resolution).

$\mathrm{mA}$, with an $\sim 8$-sec delay between each step. Each data point corresponds to a ten-point average $(3.2 \mathrm{MHz})$ of the laser noise around 97.5 MHz, followed by a subtraction of the thermal noise level. The shot-noise level was obtained by repeating the scan using manual adjustment of a halogen lamp (at 21 points distributed over the full range). Excellent linearity in the shot-noise level was obtained. A linear fit to the shot-noise trace (with thermal noise subtracted) was then used to normalize the experimental laser noise points shown on the graph.

The theoretical noise trace was calculated by adapting the theory of Ref. [8] to include the noise due to internal losses and optical losses external to the laser. Parameters used are $n_{\mathrm{sp}}=2, \quad \beta=0.5 \times 10^{-3}, \quad \tau_{\mathrm{sp}}=2 \times 10^{-9} \mathrm{~ns}, \quad \gamma_{e}=1.5 \times 10^{11}$ $\mathrm{sec}^{-1}$, and $\gamma_{0}=2.25 \times 10^{11} \mathrm{sec}^{-1}$ corresponding to the inversion parameter, fraction of spontaneous emission into the lasing mode, spontaneous emission lifetime, output coupling rate, and internal loss rate, respectively. The value of $\beta$, which is nearly two orders of magnitude larger than the value in conventional semiconductors lasers, was obtained by a fit of the power in the primary lasing mode near threshold (recorded from an optical spectrum analyzer) versus drive current. This technique has been used previously to obtain $\beta$ values for other devices [13]. Values of $\beta$ as high as $4 \times 10^{-2}$ have been obtained on similar devices (from the same wafer) using other techniques [14].

The single-mode theory is found to agree with the laser noise up to a pump rate of 1.2. Above this pump rate, the data no longer agree with the theory. This behavior can be understood by examining the optical-frequency-resolved output in an optical spectrum analyzer. Figure 3(a) shows the spectrum corresponding to a pump rate of 0.92 . Although the laser operates in only a single longitudinal mode, structure from several transverse modes are observed. The frequency splitting between these modes has been well described with a zigzag ray model [11]. The laser operation shown in Fig. 3(a) is characteristic of the region from threshold up to a pump rate of about 2 , with $20 \mathrm{~dB}$ or more separating the power in the fundamental mode at $959 \mathrm{~nm}$ from the power in the higher-order transverse modes. The noise peak near a pump rate of 2 (see Fig. 2) corresponds to the onset of the next-higher-order transverse mode $(0,1)$ at $958 \mathrm{~nm}$. Once this

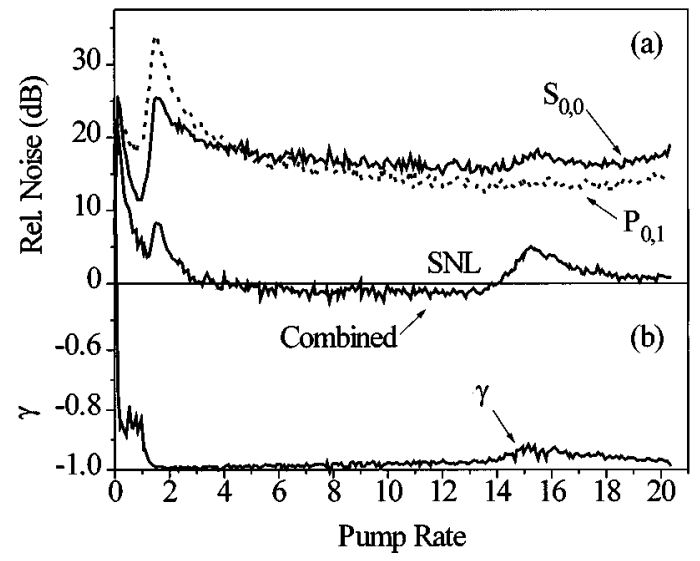

FIG. 4. (a) Polarization-resolved noise power at $97.5 \mathrm{MHz}$ vs laser pump rate. For $S_{0,0}$ the polarizer is aligned with the $(0,0)$ transverse mode, and for $P_{0,1}$ it is aligned with the $(0,1)$ mode, which is in the orthogonal polarization. For Combined the polarizer is removed. (b) The degree of correlation $-1 \leqslant \gamma \leqslant 1$ between $S_{0,0}$ and $P_{0,1}$.

mode is oscillating above its multimode threshold, the noise falls to a level below shot noise, but still $>0.6 \mathrm{~dB}$ above the single-mode theory. Figure 3(b) shows the optical frequency spectrum at a pump rate of 11 (corresponding to the data in Fig. 1). The amplitude of the $(0,1)$ mode is in fact above the fundamental, and the spectrum remains in this condition up to pump rates close to 15 . By further resolving this spectrum with a polarizer in the field, the $(0,0)$ and $(0,1)$ modes are observed to be operating in orthogonal, linearly polarized modes. Such orthogonally polarized transverse modes have been observed previously in similar devices [15]. The noise peak near a pump rate of 18 corresponds to the onset of a number of higher-order transverse modes.

The quantum correlation between the transverse modes was further explored by taking polarization-resolved scans similar to the scan in Fig. 2. The intensity noise scans (no averaging) for the polarizer aligned with the $(0,0)$ and $(0,1)$ modes, along with the noise when the polarizer is removed, are shown in Fig. 4(a). Once the $(0,1)$ mode reaches a multimode threshold, the noise on both lasing modes is more than $10 \mathrm{~dB}$ above their respective shot-noise levels. Using the scans shown in Fig. 4(a), it is possible to calculate the degree of correlation $-1 \leqslant \gamma \leqslant 1$ between the modes, as follows:

$$
\gamma=\frac{\left\langle\Delta I_{C}^{2}\right\rangle-\left(\left\langle\Delta I_{0,0}^{2}\right\rangle+\left\langle\Delta I_{0,1}^{2}\right\rangle\right)}{2 \sqrt{\left\langle\Delta I_{0,0}^{2}\right\rangle\left\langle\Delta I_{0,1}^{2}\right\rangle}}
$$

where $\left\langle\Delta I_{C}^{2}\right\rangle$ is the photocurrent power spectral density for the combined (no polarizer) spectrum, and $\left\langle\Delta I_{0,0}^{2}\right\rangle$ and $\left\langle\Delta I_{0,1}^{2}\right\rangle$ are the spectral densities for the field polarized to maximize the fundamental and $(0,1)$ mode (which are orthogonal), respectively. The numerator is equal to twice the corresponding cross-spectral density: $\left\langle\Delta I_{0,0} \Delta I_{0,1}\right\rangle$. The value of $\gamma$ for these data is shown in Fig. 4(b) as a function of drive current. The correlation is found to be -0.99 , nearly perfect, shortly after the $(0,1)$ mode goes through the threshold and gradually decreases to -0.97 at a pump rate of 14 . The mag- 
nitude of $\gamma$ actually decreases slightly from its maximum as the correlation extends below shot noise. With a straightforward subtraction of uncorrelated vacuum field contributions (taking into account individual mode efficiencies), $\gamma$ is found to most closely approach -1 near a pump rate $R=13$. However, in order to account for quantum-noise sources correctly, a quantum laser theory that includes multiple spatial and polarization modes is required. The decrease in correlation with increasing pump rate is likely due to a combination of the increase in the quantum noise associated with vacuum fluctuations from losses both internal and external to the laser (which roughly increase with the average photon number) and the gradual rise of the higher-order transverse modes.

We are not aware of a quantum laser theory that treats correlations between multiple spatial modes; however, nonclassical photon-number correlations have been observed and treated theoretically among several longitudinal modes in conventional semiconductor lasers [6]. Such correlations among modes can be understood by considering mode coupling through the homogeneously broadened gain medium. Different lasing modes receive gain from the same carrier population. Since the number of carriers is conserved in a high-efficiency device, increased recombination into one lasing mode will result in decreased recombination into the other. Thus the photon-number fluctuations in different modes become anticorrelated. In this situation, the fluctuations of the total number of output photons is still set by the noise on the laser pump (drive current) far above the threshold and thus may still be below shot noise, with the anticorrelated mode competition fluctuations canceling.

Nonlinear gain effects may also influence the mode correlations. In particular, multiwave mixing resulting in coherent modification of the gain profile has been observed to be enhanced in microcavity devices [16]. The role of such effects on the photon-number fluctuations, particularly in the quantum regime, has not yet been resolved and is an area of active research.
Either incomplete cross-mode gain saturation or unequal individual mode losses can reduce the correlation, resulting in an increase in noise on the total laser output. If crossmode gain saturation is the operative correlation mechanism in these devices, such effects are likely causes for the deviation from the single-mode theory observed in Fig. 2. Indeed, if the numerical aperture of the collimating lens is insufficient $(<0.4$ for this laser), the higher-order modes receive preferred attenuation, increasing the observed laser noise. In this work, optical scattering at the edges of the oxide confinement layers [17], which is likely to be larger for the higher-order transverse modes, could be influencing the correlation, regardless of the mode-coupling mechanism. In addition, the inhomogeneous broadening is expected to be enhanced due to strain and LO-phonon scattering, and crosstransverse mode gain saturation requires carrier thermalization over distances on the order of the mode dimensions (micrometers) and therefore may be incomplete. However, since the number of competing modes is small and the difference between the individual mode gains is large compared to the situation in conventional semiconductor lasers, the requirement of a predominantly homogeneously broadened gain medium to achieve large squeezing [6] is relaxed in these devices.

Photon-number squeezed output has been observed from a microcavity laser. This observation confirms the predictions of the high-impendance pump-noise suppression model for high-finesse, microcavity devices. Deviations from the single mode quantum-Langevin equation theory are correlated with the onset of multi-transverse-mode oscillation. Nevertheless, the noise associated with transverse-mode competition is shown to exhibit strong nonclassical correlations, which enable the observation of photon-number squeezed output.

The authors wish to thank Duncan Steel for initial discussions. This work was supported by the NSF-EPSCOR, NASA-EPSCOR, ILX Lightwave, and the US DOE under Contract No. DE-AC04-94AL85000.
[1] M. C. Teich and B. E. A. Saleh, J. Opt. Soc. Am. B 2, 275 (1985)

[2] Y. Yamamoto and N. Imoto, IEEE J. Quantum Electron. QE22, 2032 (1986); Y. Yamamoto, S. Machida, and O. Nilsson, Phys. Rev. A 34, 4025 (1986); S. Machida, Y. Yamamoto, and Y. Itaya, Phys. Rev. Lett. 58, 1000 (1987).

[3] Y. M. Golubev and I. V. Sokolov, Zh. Eksp. Teor. Fiz. 87, 408 (1984) [Sov. Phys. JETP 60, 234 (1984)].

[4] D. C. Kilper et al., Opt. Lett. 21, 1283 (1996).

[5] W. H. Richardson, S. Machida, and Y. Yamamoto, Phys. Rev. Lett. 66, 2867 (1991).

[6] W. H. Richardson and R. M. Shelby, Phys. Rev. Lett. 64, 400 (1990); S. Inoue et al., Phys. Rev. A 46, 2757 (1992); H. Wang, M. J. Freeman, and D. G. Steel, Phys. Rev. Lett. 71, 3951 (1993); J. Kitching, A. Yariv, and Y. Shevy, ibid. 74, 3372 (1995); F. Marin et al., ibid. 75, 4606 (1995)

[7] E. Goobar et al., Appl. Phys. Lett. 67, 3697 (1995).
[8] D. Kleppner, Phys. Rev. Lett. 47, 233 (1981).

[9] Y. Yamamoto, S. Machida, and G. Bjork, Phys. Rev. A 44, 657 (1991).

[10] D. L. Huffaker, D. G. Deppe, and T. J. Rogers, Appl. Phys. Lett. 65, 1611 (1994).

[11] K. L. Lear et al., Appl. Phys. Lett. 66, 2616 (1995).

[12] M. J. Freeman et al., Opt. Lett. 18, 379 (1993).

[13] F. Koyama, K. Morito, and K. Iga, IEEE J. Quantum Electron. 27, 1410 (1991).

[14] D. Kuksenkov, H. Temkin, K. Lear, and H. Hou, Appl. Phys. Lett. 70, 13 (1997).

[15] C. J. Chang-Hasnain et al., IEEE J. Quantum Electron. 27, 1402 (1991).

[16] A. E. Paul et al., Phys. Rev. A 42, 1725 (1990); C. W. Lowry et al., Phys. Rev. Lett. 71, 1534 (1993).

[17] E. R. Hegblom, D. I. Babic, and L. A. Coldren, Appl. Phys. Lett. 68, 1757 (1996). 